\title{
Caracterização química e morfológica de mistura de ureia com vermiculita encapsulada em derivado celulósico
}

\author{
Chemical and morphological description of urea mixed with vermiculite encapsulated in \\ cellulosic derivative
}

\author{
A. A. Barros ${ }^{1 *}$; R. dos $\operatorname{Santos}^{2} ;$ G. Cardoso \\ ${ }^{1}$ Programa de Pós-Graduação em Engenharia Química/Universidade Federal de Sergipe, 49100-000, São Cristóvão- \\ Sergipe, Brasil \\ ${ }_{2}^{2}$ Departamento de Engenharia Química/Universidade Federal de Sergipe, 49100-000, São Cristóvão, Brasil
}

${ }^{3}$ Departamento de Engenharia Química/Laboratório de Caracterização de Materiais/Universidade Federal de Sergipe, 49100-000, São Cristóvão-Sergipe, Brasil

*anaameliagama@gmail.com

(Recebido em 30 de janeiro de 2016; aceito em 25 de abril de 2016)

\begin{abstract}
Estudos que tenham por objetivo inibir a evaporação e a nitrificação em adubação com aplicação de fertilizantes nitrogenados são bem-vindos para a agricultura mundial, uma vez que resultam na conservação do fertilizante nitrogenado no solo e no aumento da eficiência da absorção do nitrogênio $(\mathrm{N})$ pela planta. $\mathrm{O}$ presente trabalho trata da avaliação de incorporação de ureia em vermiculita, por processo de mistura encapsulada com derivado celulósico, obtida por extrusão quanto ao seu potencial de liberação controlada de nitrogênio. O teor de ureia empregado na mistura foi de $30 \%$ em massa e a solução aquosa de derivado celulósico utilizada no encapsulamento foi de $1 \%$ em massa de carboximetilcelulose (CMC). Caracterização química e morfológica dos componentes e do produto obtido foi realizada, respectivamente por FTIR e Microscopia Eletrônica de Varredura (MEV). Os resultados das análises químicas e morfológicas mostraram que o processo de incorporação da ureia na vermiculita se deu de forma efetiva e não alterou a estrutura lamelar compacta e disposta em camadas de blocos sobrepostas, típica desse argilomineral.

Palavras-chave: produto de liberação controlada, ureia, vermiculita.
\end{abstract}

Studies that aim to inhibit evaporation and nitrification in soil fertilization with application of nitrogen fertilizers are welcome to the world agriculture, since their result is the conservation of nitrogen fertilizers in the soil and increases the absorption efficiency of nitrogen $(\mathrm{N})$ by the plant. This present study regards the incorporation of urea evaluation in vermiculite, in an encapsulated mixing process with cellulosic derivative, obtained by extrusion for their potential in a controlled nitrogen release. The urea concentration employed in the mixture was 30\% in mass and the aqueous solution of cellulosic derivative used in the encapsulation was $1 \%$ in mass carboxymethylcellulose (CMC). Chemical and morphological description of the components and the obtained product was carried out respectively by FTIR and Scanning Electron Microscopy (SEM). The results of chemical and morphological analysis showed that the urea incorporation process in vermiculite occurred effectively and did not alter the compact lamellar structure and layered overlapping blocks, typical of this mineral clay.

Keywords: controlled release product, urea, vermiculite.

\section{INTRODUÇÃO}

Estudos que tenham por objetivo inibir a evaporação e a nitrificação em adubação de solo com aplicação de fertilizantes nitrogenados são desejáveis para a agricultura, uma vez que resultam na conservação do fertilizante no solo e no aumento da eficiência da absorção do nitrogênio $(\mathrm{N})$ pela planta. Teoricamente, ao inibir a evaporação e a conversão de amônia, amônio e ureia em nitrato, se reduzem as perdas de $\mathrm{N}$ associadas ao processo de lixiviação [1].

Um fertilizante deve fornecer nutrientes necessários às plantas durante toda a etapa de crescimento, apresentar relação custo/benefício razoável e mínimo efeito adverso sobre o meio 
ambiente. No entanto, o simples uso de fertilizante não garante pleno desenvolvimento às plantas, é necessário que este seja adequado ao tipo de cultura e sua finalidade comercial. $\mathrm{O}$ aumento da eficiência no uso do fertilizante nitrogenado pode ser alcançado pela realização de mobilização, mapeamento e correção de possível acidez do solo, rotação de culturas, uso de incorporação de fertilizantes em substrato macromolecular para liberação controlada e utilização de misturas de fontes nitrogenadas. $\mathrm{O}$ sincronismo entre a época de aplicação do insumo e o período de maior exigência de $\mathrm{N}$ pelas plantas, também se constitui em importante prática no aumento da eficiência no uso do fertilizante nitrogenado, pois reduz a possibilidade de perdas de $\mathrm{N}[2,3]$.

Em solo ácido, a preocupação com a nitrificação pode ser minimizada com a aplicação de ureia incorporada ou revestida a substratos inertes, precedida de uma correção da acidez (calagem), fazendo com que o nitrogênio, proveniente da hidrólise da amônia $\left(\mathrm{NH}_{3}\right)$, fixe na forma de amônio $\left(\mathrm{NH}_{4}^{+}\right)$, que é estável [4]. A ureia é um dos fertilizantes nitrogenados mais utilizados na agricultura e que apresenta como vantagens: menor preço entre os fertilizantes, alta solubilidade, baixa corrosividade, compatibilidade com outros fertilizantes e defensivos agrícolas e alta taxa de absorção pelas plantas, e como desvantagem a possibilidade de altas perdas de $\mathrm{N}$ por volatilização de $\mathrm{NH}_{3}$ e conversão em nitrato [5].

Nesse contexto, alternativas que aumentem o desempenho de fertilizantes nitrogenados e minimizem os efeitos negativos do uso da ureia como fonte de nitrogênio são de grande importância para o agronegócio, tendo em vista o crescimento do comércio nacional e internacional de alimentos e os requisitos para o aumento de produção vinculado à qualidade exigida pelo mercado. Diante disso, buscam-se novas rotas tecnológicas para o desenvolvimento de fertilizantes nitrogenados que atendam às necessidades nutricionais das culturas desde o período inicial de crescimento até o ponto de colheita, agregando valor à produção, reduzindo custo de mão de obra e perdas de nutrientes, seja por transformações químicas ou por ação das intempéries.

Os fertilizantes de liberação controlada, principalmente os encapsulados ou recobertos, são de grande interesse devido à possibilidade de diminuição das perdas de $\mathrm{N}$ por lixiviação, volatilização e favorecimento de liberação gradual do $\mathrm{N}$ às plantas, reduzindo a necessidade de reposição periódica de sua aplicação. A extrusão é um dos mecanismos tecnológicos empregados na obtenção de produtos de liberação controlada de nitrogênio (PLCN) como processo de incorporação dos componentes a serem liberados [6,7].

Dentre os meios de produção dos fertilizantes de liberação controlada deve-se considerar o emprego da vermiculita como suporte de incorporação, devido às suas propriedades de superfície somadas aos elevados valores de área superficial específica, porosidade e carga superficial (negativa), que a torna um material adequado para o uso como adsorvente ou como carreador [8].

A produção em escala industrial da vermiculita é realizada de forma concentrada em algumas regiões no mundo e a intensidade da sua aplicação é considerada um indicador do grau de desenvolvimento tecnológico de um país [9]. Baseando-se nos relatos acima, este trabalho teve como objetivo desenvolver uma rota tecnológica para obtenção de um Produto de Liberação Controlada de Nitrogênio (PLCN) a partir da mistura de ureia com vermiculita, encapsulada em derivado celulósico, para uso em solo agrícola.

\section{MATERIAL E MÉTODOS}

\subsection{Materiais}

Para o desenvolvimento do trabalho foi utilizada como fonte de nitrogênio a ureia fornecida pela Fábrica de Fertilizantes Nitrogenados de Sergipe - FAFEN, localizada na Rodovia SE-211 km 1, Centro, Laranjeiras/SE. Como suporte de incorporação da ureia utilizou-se a vermiculita expandida comercial, oriunda do estado de Goiás e, como agente de encapsulamento a carboximetilcelulose, da Neon Comercial Ltda. 


\subsection{Preparação do PLCN}

A rota tecnológica para a obtenção do PLC se deu por meio de mistura de ureia e vermiculita, incorporada em hidrogel de carboxmetilcelulose (CMC). Antes do processamento de incorporação da ureia, a vermiculita, foi submetida à secagem, em estufa com circulação de ar a $30^{\circ} \mathrm{C}$, até peso constante. Após secagem foi preparada uma mistura de $30 \%$ de ureia com $70 \%$ de vermiculita, homogeneizada no multiprocessador Cadence, modelo MPR 860 a $100 \mathrm{rpm}$.

A obtenção do hidrogel se deu a partir da solução a 1,0\% (m/m) do agente de encapsulamento $\mathrm{CMC}$ em água deionizada a $80^{\circ} \mathrm{C}$ que após resfriada à temperatura ambiente foi adicionada lentamente à mistura de ureia-vermiculita em quantidade suficiente para promover a agregação das partículas do produto por encapsulamento. Em seguida o material foi submetido à extrusão a fim de incorporar efetivamente a ureia na vermiculita. $\mathrm{O}$ material extrudado foi seco em estufa com circulação de ar a $30^{\circ} \mathrm{C}$ até atingir peso constante.

\subsection{Caracterização da Vermiculita e do PLCN}

A vermiculita foi caracterizada quimicamente por Espectroscopia de Infravermelho com Transformada de Fourier (FTIR). Para tal, amostras foram preparadas na forma de pastilha, com mistura de vermiculita com KBr, em uma proporção de 1:100 (m/m) e grupos funcionais e ligação químicas características presentes na vermiculita foram identificados, utilizando o equipamento Thermo Scientific, Nicolet iS10. Os espectros foram obtidos aplicando 64 varreduras com resolução de $4 \mathrm{~cm}^{-1}$ e as curvas resultantes foram ajustadas utilizando o programa OriginPro $9.0^{\circledR}$.

A comprovação da incorporação da ureia no PLCN, a presença de grupos funcionais e dos tipos de ligações presentes no material em estudo foi realizada por espectrometria de FTIR, seguindo a mesma metodologia utilizada para a vermiculita pura.

A caracterização morfológica da vermiculita e do PLCN obtido foi observada por microscopia eletrônica de varredura (MEV), utilizando o aparelho Tabletop Microscope, Hitachi, modelo TM 3000 e por difração de raios X (DRX), utilizando o equipamento Bruker D8-Advance série Da Vinci com fonte de radiação $\mathrm{CuK} \alpha$. A faixa angular de varredura empregada foi de $4^{\circ}$ a $75^{\circ}$, com passo de $0,02^{\circ}$ e tempo de aquisição de 0,4 segundos.

Com a finalidade de determinar o modelo cinético de difusão do PLCN, foi realizado um estudo da quantidade de nitrogênio total liberado em água, pelo método de Kjeldahl, no Laboratório de Bromatologia do Instituto Tecnológico e de Pesquisas do Estado de Sergipe - ITPS. Os dados experimentais obtidos foram submetidos ao tratamento do mecanismo envolvido no processo, através da aplicação do modelo matemático de Korsmeyer-Peppas [10,11,12], com auxílio do software OriginPro 9.0. Esse modelo foi empregado em todo o perfil de liberação, tendo em vista que a fração liberada da substância ativa, $\mathrm{Mt} / \mathrm{M} \infty<0,6$ durante todos os noventa dias da análise.

\section{RESULTADOS E DISCUSSÃO}

Os resultados das análises espectrofotométricas por FTIR da vermiculita, ureia e PLCN são apresentados na Figura 1. Verifica-se que a vermiculita apresenta uma banda de absorção larga e intensa em $3420 \mathrm{~cm}^{-1}$ que é atribuída à deformação axial de ligação de oxigênio com hidrogênio $(\mathrm{O}-\mathrm{H})$ intermolecular presente na estrutura molecular da vermiculita e na água adsorvida [13]; a banda de número de onda $2360 \mathrm{~cm}^{-1}$ está associada ao estiramento do $\mathrm{Si}-\mathrm{H}$ [14]; outra banda característica, a de número de onda em aproximadamente $1639 \mathrm{~cm}^{-1}$ refere-se à deformação angular simétrica da água, confirmando a presença de moléculas de água entre as lamelas e moléculas de água adsorvida na superfície da vermiculita. Outra banda de intensidade absorção forte é observada no número de onda $1005 \mathrm{~cm}^{-1}$ que se refere ao estiramento assimétrico dos grupos $\mathrm{Si}-\mathrm{O}-\mathrm{Si}$ e $\mathrm{Si}-\mathrm{O}-\mathrm{Al}$ presentes nas folhas tetraédricas e octaédricas da vermiculita $[15,16]$.

As absorções observadas no número de onda $820 \mathrm{~cm}^{-1}$ e $680 \mathrm{~cm}^{-1}$ são atribuídas, respectivamente, às vibrações de deformação da ligação $\mathrm{Al}-\mathrm{OH}$, e $\mathrm{Al}-\mathrm{O}$ e a de número de onda $463 \mathrm{~cm}^{-1}$ à deformação da ligação $\mathrm{Si}-\mathrm{O}$. Ainda na Figura 1 , os espectros de infravermelho da ureia e do PLCN apresentam bandas de absorção de 3480 e $3349 \mathrm{~cm}^{-1}$ que correspondem à vibração 
assimétrica e simétrica do grupo N-H, original da ureia; a absorção da banda em torno de 1647 $\mathrm{cm}^{-1}$ está associada ao alongamento de vibração dos grupos $-\mathrm{C}=\mathrm{O}$ da ureia, comprovando a sua incorporação ao produto final.

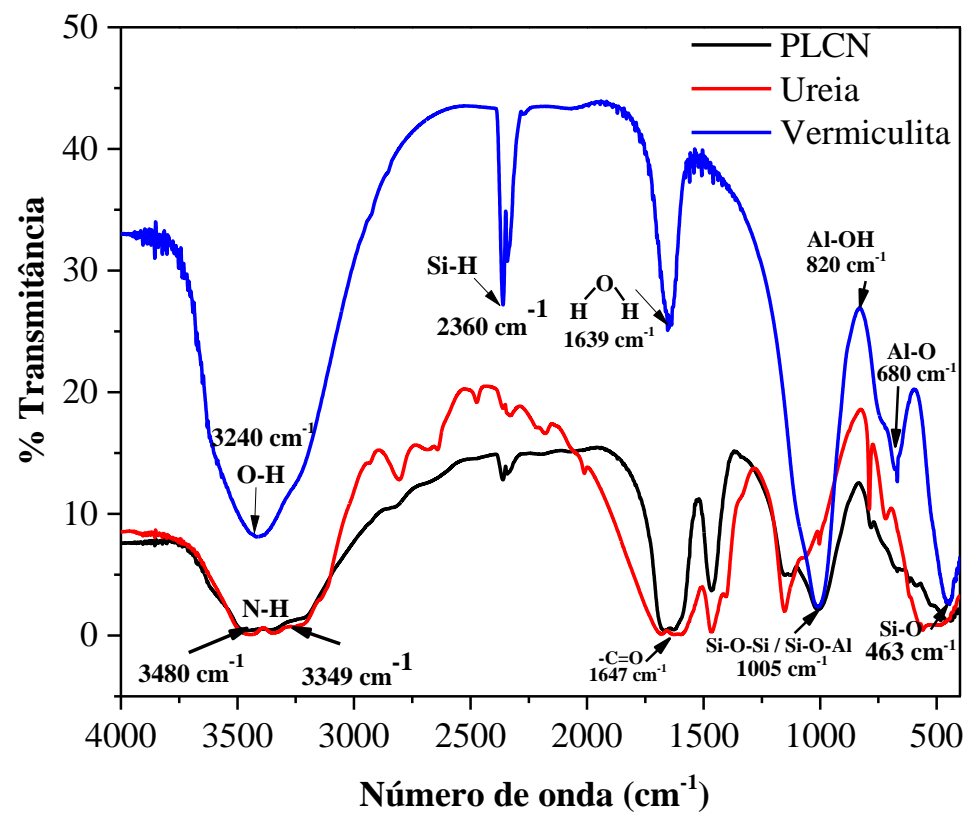

Figura 1: FTIR da vermiculita, da ureia e do PLCN (KBr)

A Figura 2 mostra a morfologia lamelar compacta sobreposta e disposta em blocos da vermiculita, típica desse argilomineral, com ampliação de 500 vezes, obtida por MEV. A Figura 3 mostra a morfologia do PLCN com a mesma ampliação da micrografia da vermiculita mostrada na Figura 2. Observa-se que esta também se apresenta de forma lamelar e estratificada [17].

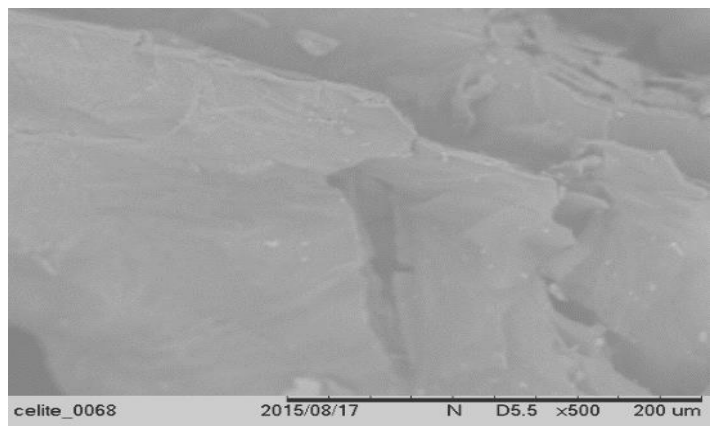

Figura 2: Micrografia por MEV da Vermiculita

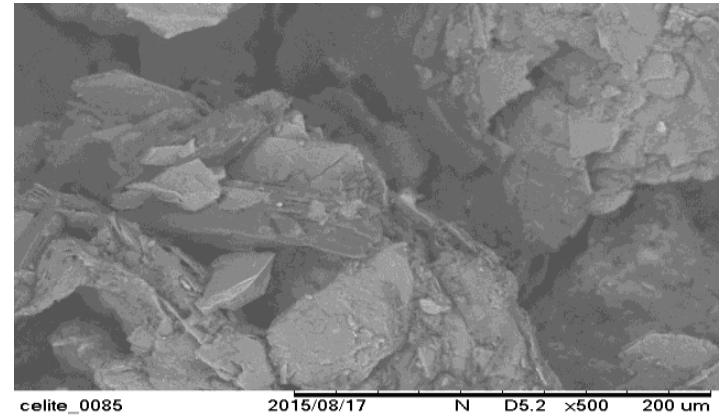

Figura 3: Micrografia por MEV do PLCN

Com a finalidade de constatar se a incorporação da ureia modificou as fases cristalinas da vermiculita, foram analisadas por DRX amostras de vermiculita e do PLCN desenvolvido. Para tal, cada amostra foi moída, para minimizar orientação preferencial dos planos cristalinos e garantir a sua homogeneidade, antes da realização da análise. Como pode ser visto nos difratogramas da Figura 4, tanto a vermiculita pura como o PLCN permanecem com estrutura organizada e cristalina. 


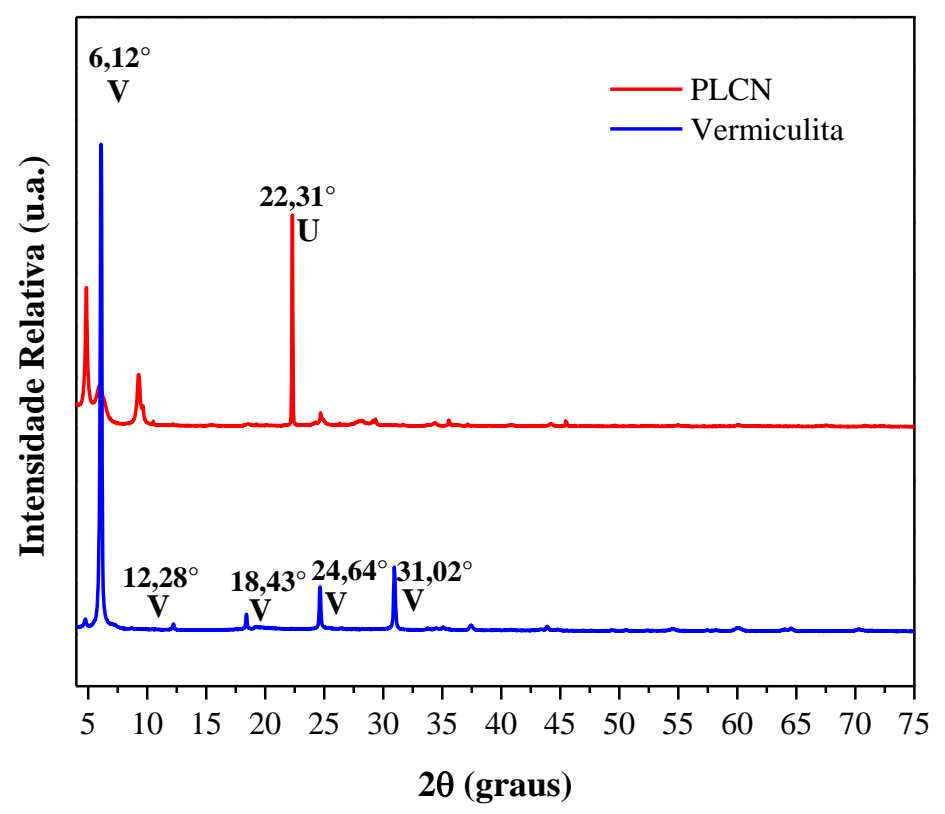

Figura 4: Difratograma da vermiculita e do PLCN

Observa-se também na Figura 4, que no difratograma da vermiculita, em azul, picos

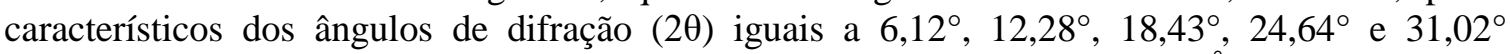
correspondem aos espaçamentos interplanares de 14,44, 7,21, 4,81, 3,61 e 2,88 Å, respectivamente, caracterizando que a amostra é constituída predominantemente por mineral vermiculita [18]. Em relação ao difratograma do PLCN, observam-se os picos de difração $6,12^{\circ}$, característico da vermiculita, e 22,31 $1^{\circ}$ que corresponde ao espaçamento interplanar de 3,99 A, típico da ureia [19].

Os resultados discutidos acima comprovam a incorporação da ureia, possibilitando a liberação controlada de $\mathrm{N}$, que é um dos nutrientes mais requeridos, sendo diretamente responsável pelo crescimento das plantas [20], e embora seu uso seja fundamental, a aplicação direta deste, sem o devido equilíbrio na dosagem, pode acarretar danos irreversíveis ao solo, tornando-o impróprio para o cultivo devido à capacidade de eutrofização. Nesse contexto, o PLCN produzido, tem destaque como alternativa para o uso na agricultura, tendo em vista que a presença da vermiculita, devido às suas propriedades, reduz a velocidade de liberação de $\mathrm{N}$ ao longo do tempo, como observado no perfil de liberação de $\mathrm{N}$ mostrado na Figura 5, podendo resultar em uma correção deste nutriente de forma eficiente e equilibrada sem danos ao solo.

O PLCN produzido neste estudo promoveu liberação lenta de $\mathrm{N}$ e o seu desempenho superior é corroborado pelos resultados obtidos por Silva et al. 2014 [21], que realizaram experimentos utilizando hidróxidos duplos lamelares sintéticos a fim de promover liberação controlada de $\mathrm{N}$, obtendo bons resultados, verificados também pelas técnicas de FTIR e DRX. Assim como Aouada et al. 2008 [22] e Geng et al. 2015 [23] que observaram a eficiência na liberação controlada de N quando incorporando fertilizantes convencionais em hidrogéis ou utilizando fertilizantes revestidos e investigando a taxa de liberação durante um período de tempo, obtendo resultados promissores, A Figura 5 mostra o perfil cinético de liberação cumulativa de nitrogênio do PLCN produzido neste trabalho, durante um período de observação de noventa dias (2160 horas) onde o modelo cinético de Korsmeyer-Peppas foi aplicado na determinação dos parâmetros cinéticos, constante da taxa de liberação (K) e expoente de liberação que caracteriza o mecanismo de transporte envolvido (n). Os dados obtidos revelam que a ureia incorporada à vermiculita, nos intervalos iniciais promove liberação rápida, no entanto, ao longo do período de verificação, a liberação ocorre de forma gradativa, caracterizando liberação lenta e eficiente de $\mathrm{N}$. 


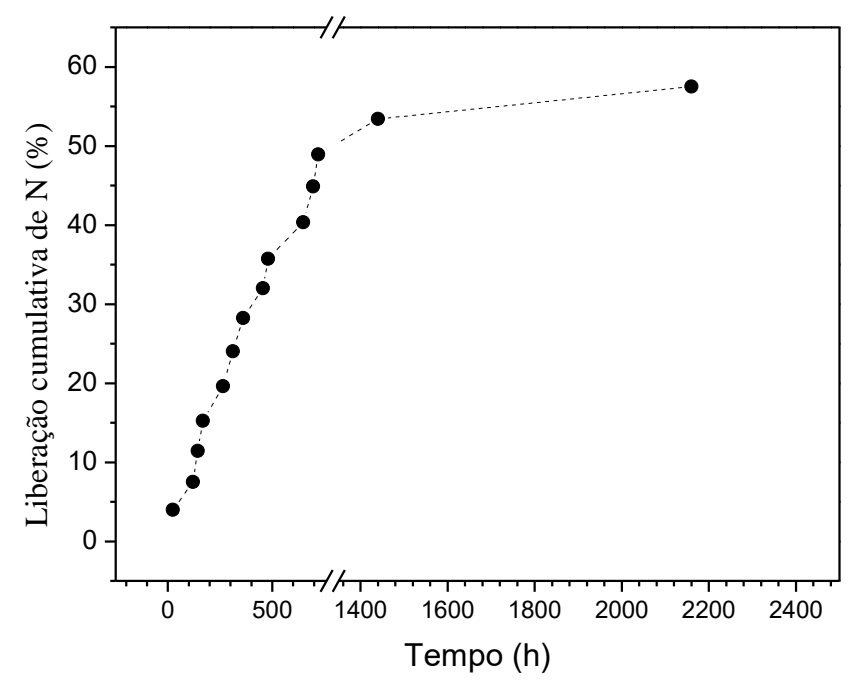

Figura 5: Perfil de liberação controlada cumulativa de nitrogênio em água do PLCN

\section{CONCLUSÃO}

A proposta de incorporar ureia à vermiculita como meio de evitar a rápida hidrólise enzimática e a volatilização da amônia foi bem sucedida, tendo em vista que o PLCN obtido forneceu liberação lenta de $\mathrm{N}$, sendo excelente alternativa como fonte principal deste nutriente para as plantas, evitando o possível risco de eutrofização do solo e baixa eficiência na fertilização. Além disso, a rota tecnológica desenvolvida nesse trabalho possibilitou a produção de um fertilizante estável, de ação prolongada e baixo custo de produção.

A morfologia lamelar compacta sobreposta e disposta em bloco da vermiculita favoreceu a incorporação da ureia na obtenção do PLCN sem alteração da sua estrutura cristalina, o que foi comprovado em todas as análises realizadas.

O processo de incorporação da ureia na vermiculita ocorreu por adsorção nas superfícies das suas lamelas e nos seus espaços intermoleculares conferindo ao PLCN boas características para atuar como produto de liberação controlada de componentes fertilizantes em solo agrícola.

\section{AGRADECIMENTOS}

Os autores agradecem ao Programa de Pós-graduação em Engenharia Química da Universidade Federal de Sergipe, ao Instituto Tecnológico e de Pesquisas do Estado de Sergipe - ITPS e ao Departamento de Química da Universidade Federal de Sergipe pela cooperação, apoio e suporte durante todo o período de desenvolvimento deste estudo.

\section{REFERÊNCIAS BIBLIOGRÁFICAS}

1. Trinh TH, Kushaari K, Shuib AS, Ismail, L, Azeem,B. Modelling the release of nitrogen from controlled release fertiliser: Constant and decay release. Biosyst Eng. 2015 Fev;130:3442,doi:10.1016/j.biosystemseng.2014.12.004.

2. Civardi EA, Silveira Neto AN, Ragagnin VA, Godoy ER, Brod E. Ureia de liberação lenta aplicada superficialmente e ureia comum incorporada ao solo no rendimento do milho. Pesq Agropec Trop, Goiânia 2011 Jan/Mar;41(1):52-59, doi:10.5216/pat.v41i1.8146.

3. Jua X-T, Guang-Xi X, Chena X-P, Zhang S-L, Zhang L-J, Liu X-J, Cui Z-L, Yin B, Christie P, Zhu Z-L, Zhang F-S. Reducing environmental risk by improving $\mathrm{N}$ management in intensive Chinese agricultural systems. Proc Natl Acad Sci U.S.A. 2009 Mar;106(9):3041-3046, doi:10.1073/pnas.0813417106. 
4. Stafanato JB, Goulart R S, Zonta E, Lima E, Mazur N, PereiraCG, Souza HN. Volatilização de amônia oriunda de ureia pastilhada com micronutrientes em ambiente controlado. Rev Bras Ciênc Solo. 2013 Mar;37(3):726-732, doi:10.1590/S0100-06832013000300019.

5. Martins IS, Cazetta J, Fukuda AJF. Condições, modos de aplicação e doses de ureia revestida por polímeros na cultura do milho. Pesq Agropec Trop, Goiânia 2014 Jul/Set;44(3):271-279, doi: 10.1590/S1983-40632014000300010.

6. Bialleck S, Rein H. Preparation of starch-based pellets by hot-melt extrusion. Eur J Pharm Biopharm. 2011 Out;79(2):440-448, doi: 10.1016/j.ejpb.2011.04.007.

7. Vervaet C, Baert L, Remon JP. Extrusion-Spheronisation a Literature Review, Int J Pharm. 1995 Mar;116(2):131-146, doi: 10.1016/0378-5173(94)00311-R.

8. Ugarte JFO, Sampaio JA, França SCA. Ministério da Ciência e Tecnologia, Centro de Tecnologia Mineral - CETEM, Coordenação de Processos Minerais - COPM, Vermiculita, Capítulo 32. CT2005-143-00: Comunicação Técnica elaborada para Edição do Livro Rochas \& Minerais Industriais: Usos e Especificações; Rio de Janeiro, 2005. p. 677-698.

9. Brasil, Ministério de Minas e Energia - MME, Secretaria de Geologia, Mineração e Transformação Mineral - SGM. Do Relatório Técnico: Perfil da Vermiculita. J. Mendo Consultoria, através do Contrato n 48000.003155/2007-17: Desenvolvimento de Estudos para Elaboração do Plano Duodecenal (2010 2030) de Geologia, Mineração e Transformação Mineral. 2009.

10. Korsmeyer RW, Gurny R, Doelker E, Buri P, Peppas NA. Mechanisms of solute release from porous hydrophilic polymers. Int J Pharm. 1983 Mai;15(1): 25-35, doi:10.1016/0378-5173(83)90064-9.

11. Ritger PL, Peppas NA. A simple equation for description of solute release: II. Fickian and anomalous release from swellable devices. J Control Release. 1987 Jun;5(1):37-42, doi:10.1016/01683659(87)90035-6.

12. Siepmann J, Peppas NA. Mathematical modeling of controlled drug delivery. Adv Drug Deliv Rev. 2001 Jun;48(2-3):137-138, doi: 10.1016/S0169-409X(01)00111-9.

13. Silverstein RM, Webster FX, Kiemle DJ. Identificação Espectrométrica de Compostos Orgânicos. $7^{\mathrm{a}}$ ed. Rio de Janeiro: LTC, 2006. 490 p.

14. Ferraro JR, Krishnan K. Practical Fourier Transform Infrared Spectroscopy: Industrial and laboratory chemical analysis. 1st ed., San Diego - CA, Academic Press, Inc., 1990. 519 p.

15. Nakamoto K. Infrared and Raman spectra of inorganic and coordination compounds. $4^{\mathrm{a}}$ ed. New York: John Wiley and Sons, 1986. 484 p.

16. Lin-Vien D, Colthup NB, Fateley WG, Grasselli JG. The Handbook of Infrared and Raman Characteristic Frequencies of Organic compounds. $1^{\text {a }}$ Ed. New York Boston: Academic Press, 1991. 503 p.

17. Barabaszová KČ, Valášková M. Characterization of vermiculite particles after different milling techniques. Powder Technol. 2013 Mai;239:277-283, doi:10.1016/j.powtec.2013.01.053.

18. Bergaya F, Lagaly G. Handbook of clay science. 2a ed. Vol. 5A, Newnes, Elservier, 2013. 1197 p.

19. Nardi SQW, Teixeira SD, Parabocz CRB. Incorporação de fonte de nitrogênio em partículas de PVA e alginato de sódio e estudo da influência de ciclos de congelamento/descongelamento na caracterização do produto. Polímeros. 2015; 25(6):606-613, doi.org/10.1590/0104-1428.1967.

20. Porto JS, Amorim YF, Rebouças TNH, Lemos OL, Luz JMQ, Costa RQ. Índice SPAD e crescimento do tomateiro em formação de diferentes fontes e doses de nitrogênio. Scientia Plena 2014 Out;10(11):110203.

21. Silva V, Kamogawa MY, Marangoni R, Mangrich AS, Wypych F. Hidróxidos duplos lamelares como matrizes para fertilizantes de liberação lenta de nitrato. R. Bras. Ci. Solo. 2014 Fev;38(1):272-277, doi: 10.1590/S0100-06832014000100027.

22. Aouada FA, Moura MR, Menezes EA, Nogueira ARA, Mattoso LHC. Síntese de hidrogéis e cinética de liberação de amônio e potássio. Rev. Bras. Ciênc. Solo [online]. 2008 Mai;32(4):1643-1649, doi.org/10.1590/S0100-06832008000400029.

23. Geng J, Ma Q, Zhang M, Li C, Liu Z, Lyu X, Zheng W. Synchronized relationships between nitrogen release of controlled release nitrogen fertilizers and nitrogen requirements of cotton. Field Crop Res. 2015 Set;184:09-15, doi: 10.1016/j.fcr.2015.09.001. 